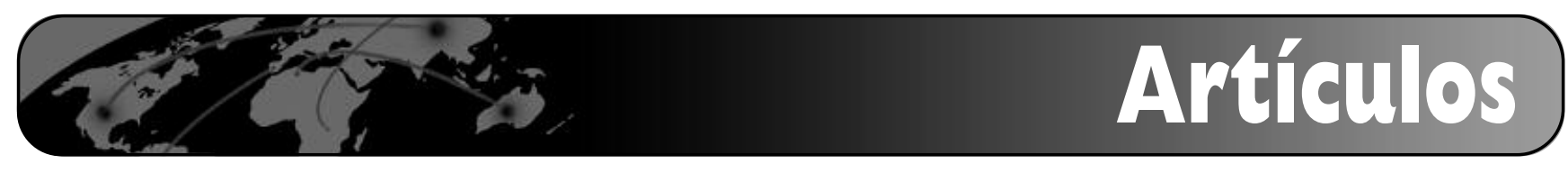

\title{
Intranets: medición y valoración de sus beneficios en las organizaciones
}

\author{
Por Daniel Pérez González y Pedro Solana González
}

\begin{abstract}
Resumen: Se muestra cómo las características del entorno empresarial actual, en el que destacan la importancia del conocimiento y los recursos humanos, deberían ser propicias para la implantación de herramientas tecnológicas como las intranets. Sin embargo, esto no sucede así en las pequeñas y medianas empresas (pymes) de España. Una de las causas que motivan esta baja adopción es la dificultad para los gestores de valorar y medir los beneficios generados por dichas tecnologías dentro de las organizaciones. En este sentido, se cuestionan los métodos tradicionales de valoración económica

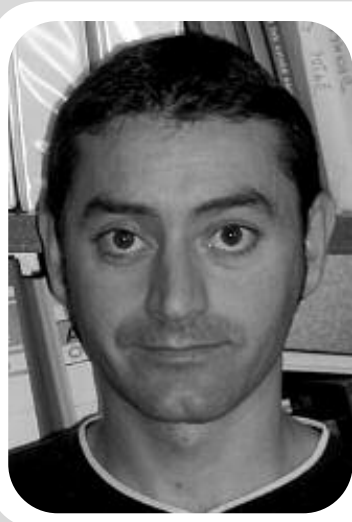
Daniel Pérez González, diplomado en ciencias económicas y empresariales, licenciado en administración y dirección de empresas y doctor en administración de empresas por la Universidad de Cantabria. Profesor de sistemas de información y miembro del grupo I+D+i de informática de gestión de dicha universidad. Ha participado como investigador del proyecto Cicyt "Diseño e implantación de un sistema de ayuda a la toma de decisiones para la gestión de la empresa" 2003-2005. Miembro de la Academia Europea de Dirección y Economía de la Empresa y colaborador del Institut for Market Research de Kiel (Alemania).
\end{abstract} y se presenta como ejemplo de valoración el análisis de los beneficios que las intranets aportan a 80 pymes del sector de las tecnologías de la información y la comunicación (TIC), mediante una metodología de medición que combina indicadores cualitativos con cuantitativos. Los resultados obtenidos muestran la contribución notable de las intranets a las organizaciones.

Palabras clave: Intranets, Valoración económica, Recursos humanos, Gestión del conocimiento, Indicadores de gestión.

Pedro Solana González, licenciado en informática por la Universidad Politécnica de Cataluña (UPC), magíster en ciencias de la computación y doctor ingeniero industrial (Univ. de Cantabria). Profesor de sistemas de información y diseño de bases de datos y miembro del grupo 1+D+i de informática de gestión de la Univ. de Cantabria. Ha participado y dirigido diversos proyectos de investigación sobre gestión documental, innovación y mejora de procesos, gestión de la experiencia operativa y sistemas de gestión integrados para Nuclenor (Central Nuclear de Sta. $M^{a}$ de Garoña), así como para Solvay Química en el área de la gestión de proyectos y workflow. Ha participado como investigador del proyecto Cicyt "Diseño e implantación de un sistema de ayuda a la toma de decisiones para la gestión de la empresa" en el período 2003-2005.

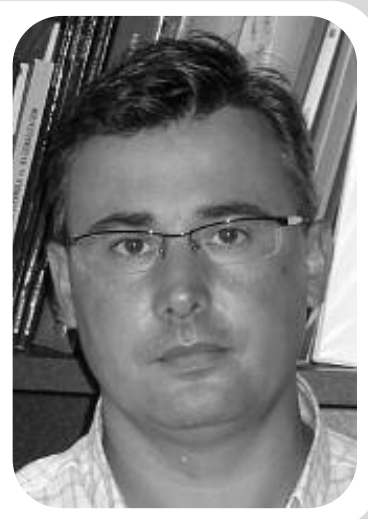

Title: Intranets: measurement and valuation of their benefits in organisations

Abstract: This work shows how the characteristics of the current business environment, in which knowledge and human resources are considered of high importance, should be propitious for implanting technologies like intranets. Nevertheless, this is not happening in Spain's small and medium-sized enterprises (SMEs). One of the causes of the low rate of adoption is the difficulty for managers to value and measure the benefits generated by intranets inside the organisations. With the traditional methods of economic valuation being questioned, we present the example of an analysis of benefits that intranets generated for 80 SMEs of the ICT sector, carried out by means of a methodology of measurement that combines qualitative and quantitative indicators. The results obtained show the positive contribution of intranets to these organisations.

Keywords: Intranets, Economic valuation, Human resources, Knowledge management, Management indicators.

Pérez González, Daniel; Solana González, Pedro. «Intranets: medición y valoración de sus beneficios en las organizaciones». En: El profesional de la información, 2006, septiembre-octubre, v. 15, n. 5, pp. 331-341. 


\section{Introducción}

El actual entorno definido como global, en cuanto a que personas y empresas pueden actuar y realizar sus actividades prácticamente en cualquier parte del mundo, se ha desarrollado en gran medida gracias a las tecnologías de la información y la comunicación (en adelante las denominaremos TIC) y su uso intensivo en lo social y empresarial. Las TIC, hoy presentes en todos los ámbitos de la actividad humana, han dado lugar a escenarios abiertos y muy dinámicos, con intercambios continuos de información relativamente a bajo coste, lo que ha supuesto para las organizaciones trasladar el problema e importancia de la adquisición de información (década de los 80) a, en la actualidad, la adecuada gestión e interpretación de dicha información, es decir al conocimiento.

Cuestiones como la globalización de los mercados y la reciente ampliación de la Unión Europea facilitan la deslocalización industrial hacia regiones de bajos costes laborales. Esta situación obliga a nuestras economías, y en última instancia a nuestras empresas, al desarrollo de productos y servicios de mayor valor añadido, evolucionando hacia modelos en los que la importancia de los procesos industriales es reemplazada por la relevancia del procesamiento de la información y el conocimiento como claves económicas.

De esta forma la competitividad de las organizaciones se ve comprometida por dos aspectos interrelacionados: el primero, un uso intensivo y racional de las TIC que cree valor para la organización y favorezca el segundo aspecto, el conocimiento, recurso fundamental de las organizaciones.

Es precisamente en un contexto como el que hemos descrito donde adquieren mayor importancia herramientas tecnológicas como las intranets. Pueden definirse como una red de información interna circunscrita a un número limitado de usuarios, configurada sobre una red de clientes-servidor tcp/ip, con navegadores y un servidor web, y un conjunto de software y hardware que permite crear y acceder a datos hipermedia, publicar páginas web, establecer comunicaciones por vía telemática, compartir información en tiempo real, etc. (O'Brien, 2001). Como indican Marcus y Watters (2002) internet e intranet se basan en la misma tecnología siendo su principal diferencia el acceso y propiedad de la información, lo que da lugar a una de las características más importantes de las intranets, el permitir diferentes contenidos y niveles de acceso en función de los distintos roles de usuarios definidos en la organización (O'Brien, 2001).

Los servicios que se pueden ofrecer son múltiples y diversos y dependerán en gran medida de la actividad y estrategia de la organización. Pero por su defini- ción y composición puede permitir entre otras las siguientes funciones:

- Canalizar y soportar la información, documentación y conocimientos necesarios para las actividades de los empleados, incrementando su productividad.

- Definir y transmitir la cultura de la empresa, al reflejar los valores de la misma en su diseño y contenidos.

- Mostrar la imagen que los empleados poseen de la empresa, puesto que ellos la construyen al utilizarla, actualizar sus contenidos y personalizarla según sus necesidades.

- Gestionar el trabajo colaborativo, incluso de trabajadores geográficamente dispersos (teletrabajo).

-Promover la comunicación y participación del personal.

-Facilitar la formación de los empleados, el aprendizaje organizacional y los procesos de gestión de conocimiento.

Son precisamente las características y posibilidades de uso las que han dado lugar al reconocimiento de su importancia como instrumentos de gestión (tanto de información como de conocimiento) e incluso de personas. De hecho, la Comisión Europea considera su posesión como uno de los indicadores para medir la evolución y grado de cumplimiento, en el apartado de empresa, del programa $e$-Europe 2005 y su evolución hacia la sociedad de la información (Com, 2002).

\section{«Las pymes están muy lejos de beneficiarse de una mejora en su competitividad a través del empleo de las TIC en general y de las intranets en particular»}

Sin embargo, a pesar de este reconocimiento las estadísticas muestran que la implantación en las empresas españolas es muy reducida. Así, según los datos del Instituto Nacional de Estadística (INE, 2001 a 2004), en España el porcentaje de compañías que disponía de una en el año 2001 era de $23,3 \%$ siendo en 2002 del $29,5 \%$, en 2003 del $32,6 \%$ y en 2004 del $31,4 \%$, lo que supone un incremento en cinco años del $34,8 \%$, pequeño en comparación, por ejemplo, con la adopción de software de gestión empresarial orientado más a producción y control de gestión, y menos a distribuir y compartir información y conocimiento, como los enterprise resource planning (Erp) y los customer relationship management (Crm), presentes en el 70\% de nuestras empresas (Sieber; Valor, 2005). 
Además es importante destacar que el porcentaje de empresas que disponen de intranet oscila notablemente en función de su tamaño, reduciéndose del $77 \%$ de las grandes organizaciones al $27,5 \%$ de la pymes. En este sentido, los datos muestran que las grandes empresas tienen la lección tecnológica estudiada. Sin embargo, las pequeñas y medianas empresas, que no olvidemos según los datos de la Dirección General de Política de la Pequeña y Mediana Empresa (Dgpyme, 2005) constituyen y sustentan el $99 \%$ del tejido empresarial tanto de la Unión Europea como de España, están muy lejos de beneficiarse de una mejora en su competitividad a través del empleo de las TIC en general y de las intranets en particular, como refleja el gráfico de inversión media de las pymes en TIC, en el que se destaca que entorno al $40 \%$ de dichas empresas invierten por término medio menos de 1.500 euros anuales (ver gráfico 1).

Las razones de esta brecha o diferencial tecnológico entre grandes y pymes son diversas $y$, pese a que generalmente se atribuye a la menor disponibilidad de recursos económicos que tienen las de menor tamaño, la realidad es que este factor no es el único, como señala el estudio de la fundación Red.es y el Ministerio de Industria, Turismo y Comercio (2005).

En línea con lo anterior, hemos obtenido la opinión de 80 gerentes de pymes del sector TIC, que identifican las principales barreras a la inversión en una intranet: dificultad para medir si es rentable y saber qué impacto tiene en los resultados económicos de la empresa. En este trabajo se analiza la problemática en la medición de los beneficios que las intranets generan en las organizaciones, y se presenta como ejemplo la metodología seguida en el análisis de los beneficios generados por la intranet en las empresas de la muestra.

\section{Problemática en la medición y valoración de los beneficios}

En primer lugar se debe señalar que las actividades de valoración de activos y medición de beneficios en las instituciones siguen un férreo patrón marcado por la contabilidad y la economía financiera. En cuanto a la valoración contable de las intranets, las actuales aplicaciones contables que derivan de una etapa económica industrial (caracterizada por el pleno predominio del capital y los activos tangibles como recursos fundamentales) muy diferente a la actual economía de servicios y conocimiento, no son válidas para reflejar el valor real generado por los activos intangibles (Peters, 1990; Lev; Sarta; Sougianis, 1999; Lev, 2001; Cañibano, 2005). De hecho, la contabilidad tiene entre sus criterios fundamentales la depreciación y amortización, que dan por supuesto la pérdida progresiva en el tiempo del valor de una inversión por su uso y ob-

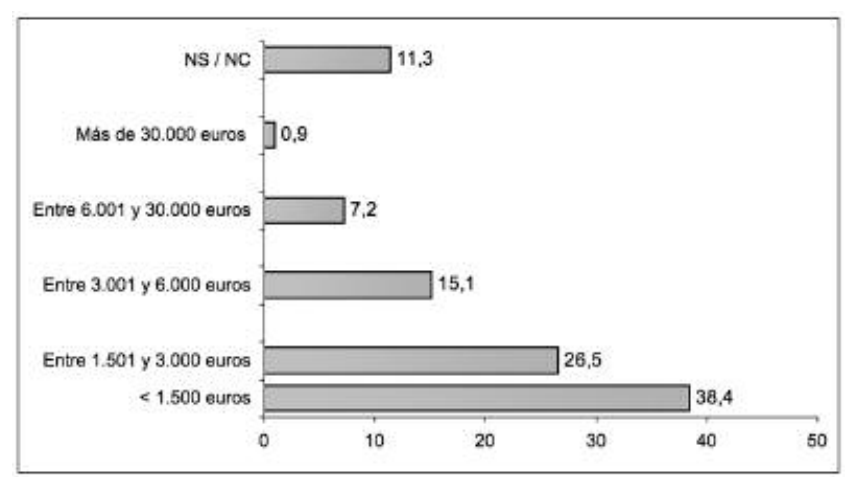

Gráfico 1. Inversión media de la pymes españolas en nuevas tecnologías 2005

Fuente: Ministerio de Ciencia y Tecnología

solescencia. Algo contrario a lo que sucede con una intranet que, por sus características propias, lo normal es que su valor aumente cuanto mayor sea su uso y contenidos. Por tanto, los criterios contables si bien son obligatorios para cumplir con la normativa mercantil, no son idóneos para aplicar en las intranets.

Respecto a la decisión de invertir en una intranet y la medición de sus beneficios priman, en general, la aplicación de criterios económicos y financieros, entre los que destacan el retorno de la inversión (ROI) y el valor actual neto (VAN) de los flujos monetarios que la inversión genera (Mahmood; Mann, 1993; Escobar, 1997; Im; Dow; Grover, 2001; Chen, et al., 2006). Estos métodos básicamente requieren identificar, individualizar y cuantificar monetariamente los beneficios que genera concretamente cada inversión. Sin embargo las intranets, desde la visión empresarial, son consideradas como elementos de infraestructura y apoyo a las actividades de la organización (Bueno, 2000; Kaplan; Norton, 2001; OCDE, 2004; Sieber; Valor, 2005), lo que supone su participación simultánea en distintos procesos de negocio que no tienen por qué estar relacionados con aspectos directamente cuantificables.

En consecuencia, cuando la naturaleza de la inversión y sus efectos son de base intangible los métodos tradicionales de selección y valoración de inversiones presentan dificultades para ser puestos en práctica debido a:

- Considerar la rentabilidad sólo en términos económicos es una visión de corto plazo, que penaliza el análisis en inversiones que tienen carácter estratégico, como es el caso de la intranet.

- La cuantificación monetaria, a priori, de los beneficios o retornos que generará deja de ser objetiva y se convierte en una opinión.

- La valoración real de los beneficios generados debe ser ex post, cuando se encuentre ya en funcionamiento. Además se ha de tener en cuenta que los be- 
neficios producidos son en muchos casos intangibles y se generan de manera indirecta: disponer de un mejor y más económico flujo de comunicación en la empresa, conocimiento compartido, etc.

- La participación simultánea de la intranet en distintos procesos y su comportamiento sinérgico dificultan a los gestores la medición y asignación individualizada de los beneficios, requisitos que exigen los criterios financieros.

\section{«Los criterios contables si bien son obligatorios para cumplir con la normativa mercantil, no son idóneos para aplicar en las intranets»}

Asimismo, la dinámica actual de las organizaciones en las que todo está interrelacionado implica una mayor complejidad en la definición y representación de la variable rendimiento (Brynjolfsson; Hitt, 1996; Paños, 2000; Standfield, 2002), que requiere para su determinación de múltiples criterios, ya no sólo cuantitativos como rentabilidad, beneficios netos y márgenes sobre ventas, sino también cualitativos relacionados con los activos intangibles (Stewart, 1997, 2001; Tissen, et al., 2000; Cañibano; Sánchez, 2004).

En esta línea, los empresarios deben ser conscientes de la necesidad de realizar análisis desde distintas perspectivas: económicas y no económicas, y establecer mecanismos de medición que muestren, tanto los efectos que la intranet tiene en aquellos procesos cualitativos que la propia organización considera claves para su gestión, como la repercusión en las variables económicas.

\section{Evaluación de la contribución de la intranet a la generación de valor, un ejemplo en pymes tecnológicas}

A continuación se presentan los resultados de una investigación ${ }^{1}$ centrada en analizar los beneficios generados por las intranets en 80 pymes del sector de las TIC. La elección de este área y tipo de organizaciones viene determinada tanto por la importancia creciente del sector dentro de las economías modernas, convirtiéndose en estratégico (OCDE, 2004; EITO, 2005; Comisión Europea, 2005), como por ser uno de los que tienen mayor potencial en la economía europea y española (Comisión Europea, 2002; Ontiveros, 2005). Y se ha considerado oportuno enfocar este trabajo hacia las pymes puesto que como ya se ha comentado son la base de nuestro tejido empresarial.
Las empresas objeto de estudio se ajustan a la definición de pyme establecida por la Comisión Europea ${ }^{2}$ (DOCE, 2003). Además se caracterizan por tener ubicada su sede principal en las comunidades de Cataluña y Cantabria, así como por presentar como principal fuente de ingresos el desarrollo de software, que por término medio representa el $58 \%$ de la cifra de negocio, seguido de la venta de equipamiento y material informático $(24,8 \%)$ y de actividades de consultoría, asesoramiento y mantenimiento $(17,2 \%)$. Tienen entre 10 y 78 empleados, con un número medio de 16 personas en plantilla, y en su cualificación predominan los titulados universitarios $-57,8 \%$ del total: $36,6 \%$ ingenieros o licenciados, y $21,2 \%$ diplomados o ingenieros técnicos-, seguido de formación profesional $(38,9 \%)$ y de un número reducido de personal no especializado $(3,4 \%)$. La formación de los dos grupos (empresas con intranet y sin ella) se hizo tomando como criterio el que dispusieran o no de una intranet operativa durante los últimos tres años previos al estudio (2001 a 2004) puesto que, aunque no existe consenso en la literatura en cuanto al período temporal que tiene que transcurrir para considerar una tecnología consolidada dentro de una organización, sí hay una tendencia a utilizar períodos de entre 2 y 5 años (Miñana, 2001; Brynjolfsson; Hitt, 2003; OCDE, 2004). El resultado final ha sido que 33 compañías (41\%) componen el grupo con intranet.

Para determinar qué tipo de efectos, positivos o negativos, ejercen las intranets se hizo una investigación empírica apoyada en métodos cualitativos (reuniones de grupo y entrevistas en profundidad) y cuantitativos (encuesta, recogida de datos y su tratamiento estadístico) que se efectuó durante los meses de enero-agosto de 2005.

De acuerdo con lo expuesto sobre la insuficiencia de los métodos de valoración tradicionales y la complejidad de la variable rendimiento, se optó por realizar una aproximación multidimensional de tipo cualitativo y cuantitativo, que permitiera conocer los efectos de las intranets en los elementos reconocidos como críticos y distintivos para el éxito de la organización. En este caso, las empresas señalaron como aspectos clave los recursos humanos y el conocimiento. Además, dado que por definición las intranets son herramientas de comunicación y coordinación, de conformidad con la opinión de los gerentes se seleccionó como variable económica a estudiar el comportamiento en el tiempo de los costes de coordinación de las organizaciones.

Para cada uno de los factores críticos fueron seleccionados unos indicadores consensuados con los gerentes de las empresas, y para recoger sus opiniones se elaboraron preguntas en forma de escalas likert $^{3}$ que 


\begin{tabular}{|c|l|l|l|l|l|l|l|l|}
\hline$I m R h 1$ & $\begin{array}{l}\text { Los trabajadores se identifican con los } \\
\text { valores y objetivos de la empresa. }\end{array}$ & 1 & 2 & 3 & 4 & 5 & 6 & 7 \\
\hline$I m R h 2$ & $\begin{array}{l}\text { Los trabajadores muestran entusiasmo } \\
\text { e implicación en sus trabajos. }\end{array}$ & 1 & 2 & 3 & 4 & 5 & 6 & 7 \\
\hline$I m R h 3$ & $\begin{array}{l}\text { Es frecuente que los empleados } \\
\text { sugieran mejoras. }\end{array}$ & 1 & 2 & 3 & 4 & 5 & 6 & 7 \\
\hline
\end{tabular}

Tabla 1. Escala formada como indicador de la implicación de los recursos humanos $l=$ total desacuerdo, $7=$ total acuerdo

\begin{tabular}{|c|c|c|c|c|}
\hline $\begin{array}{l}\text { Utilización de } \\
\text { la intranet }\end{array}$ & \multicolumn{2}{|c|}{ (Medias) ImRh1 } & $\operatorname{ImRh2}$ & $\operatorname{ImRh3}$ \\
\hline Sí & \multicolumn{2}{|c|}{5,15} & 6,00 & 5,61 \\
\hline \multirow[t]{4}{*}{ No } & \multicolumn{2}{|l|}{3,87} & 3,87 & 4,00 \\
\hline & Valor de la $F$ & 3,30 & 22,51 & 9,79 \\
\hline & Significatividad & $0,085 * *$ & 0,000* & $0,006 *$ \\
\hline & \multicolumn{4}{|c|}{$\begin{array}{l}\text { * Significatividad al nivel del } 99 \% \\
* * \text { Significatividad al nivel del } 95 \%\end{array}$} \\
\hline
\end{tabular}

Tabla 2. Anova posesión de intranet y atributos de la implicación de los recursos humanos

fueron sometidas a un análisis de fiabilidad mediante el Alpha de Cronbach ${ }^{4}$. Una vez obtenidos los indicadores y comprobada la fiabilidad de las escalas, para cada factor clave fueron generados modelos de análisis de la varianza $\left(\right.$ anova $\left.^{5}\right)$ que, a partir de la división de las empresas de la muestra en dos grupos (con posesión de intranet y sin ella) permiten comprobar la existencia de efectos estadísticamente significativos entre la utilización de una intranet (variables independientes de los contrastes), y los indicadores y escalas de cada uno de los factores (variables dependientes de los contrastes).

En el siguiente apartado se recogen y comentan los resultados obtenidos en cada uno de los factores clave mediante unas tablas que muestran, para cada grupo de empresas y en cada ítem de la escala, los valores medios, el valor del estadístico $F$ de Snedecor ${ }^{6}$ y la significatividad ${ }^{7}$ del contraste.

\section{Resultados y discusión}

\subsection{Efecto de la utilización de la intranet sobre} los recursos humanos

La literatura y los profesionales de empresa coinciden en señalar que los recursos humanos son uno de los factores determinantes para el éxito y excelencia de cualquier organización. Con objeto de realizar un análisis exhaustivo de los efectos que las intranets pueden tener sobre los recursos humanos, este factor se desdo-

\begin{tabular}{|c|l|l|l|l|l|l|l|l|}
\hline SatiRh1 & $\begin{array}{l}\text { Los empleados que finalizan voluntariamente } \\
\text { su relación con la empresa antes de fin de } \\
\text { contrato son muchos y cada vez más. }\end{array}$ & 1 & 2 & 3 & 4 & 5 & 6 & 7 \\
\hline SatiRh2 & $\begin{array}{l}\text { Los empleados están sujetos a estrés } \\
\text { laboral. }\end{array}$ & 1 & 2 & 3 & 4 & 5 & 6 & 7 \\
\hline SatiRh3 & $\begin{array}{l}\text { El absentismo laboral se ha incrementado en } \\
\text { los últimos años. }\end{array}$ & 1 & 2 & 3 & 4 & 5 & 6 & 7 \\
\hline
\end{tabular}

Tabla 3. Escala formada como indicador de la satisfacción de los recursos humanos $l=$ total desacuerdo, $7=$ total acuerdo

\begin{tabular}{|c|c|c|c|c|}
\hline $\begin{array}{c}\text { Utilización de } \\
\text { la intranet }\end{array}$ & \multicolumn{2}{|c|}{ (Medias) SatiRh1 } & SatiRh2 & SatiRh3 \\
\hline Sí & \multicolumn{2}{|l|}{1,30} & 2,76 & 1,23 \\
\hline No & \multicolumn{2}{|l|}{2,87} & 3,75 & 3,00 \\
\hline \multirow{3}{*}{$7 v 0$} & Valor de la $F$ & 10,67 & 1,74 & 12,11 \\
\hline & Significatividad & $0,004^{*}$ & 0,202 & $0,003 *$ \\
\hline & \multicolumn{4}{|c|}{ * Significatividad al nivel del 99\% } \\
\hline
\end{tabular}

Tabla 4. Anova posesión de intranet y atributos de la satisfacción de los recursos humanos 
bla en dos conceptos distintos pero vinculados, por un lado la implicación con la organización de los recursos humanos, y por otro, su satisfacción en la organización, realizando un estudio individualizado para cada uno de los conceptos.

\subsubsection{Implicación de los recursos humanos de la empresa}

El análisis se realizó mediante un modelo anova para cada uno de los atributos que componen el concepto de implicación (tabla 1) (Powell; Dent-Micallef, 1997).

La tabla 2 muestra cómo, para los 3 atributos, el grupo de empresas que dispone de intranet presenta un mayor nivel de implicación del personal, alcanzando significatividad estadística.

Por consiguiente, existe para las empresas objeto de estudio una relación positiva entre la posesión de intranet y el logro de mejores resultados en la implicación de sus recursos humanos, al facilitar una mayor identificación de los empleados con los valores de la compañía, y favorecer la participación tanto activa como positiva de los empleados en la mejora de la organización.

\subsubsection{Satisfacción de los recursos humanos de la empresa}

Como en el caso anterior, el análisis de la existencia de un efecto positivo entre la utilización de intranets y la satisfacción de los empleados se realiza mediante un modelo anova para cada uno de los atributos que componen el concepto de satisfacción (Powell; Dent-Micallef, 1997).

En este caso, en la tabla 4 se observa descriptivamente que su utilización tiene una relación positiva con todos los ítemes que integran la satisfacción del empleado. Si bien la relación es estadísticamente significativa sólo con dos de ellos -SatiRhl, relativo a la finalización voluntaria del contrato de trabajo por parte del empleado, y SatiRh3, que representa el incremento del absentismo laboral-, no se alcanzó significatividad estadística en SatiRh2, posiblemente debido al reducido tamaño muestral que exige que la distancia estadística entre medias sea muy alta, lo que dificulta la obtención de significatividad.

No obstante, la dirección de las medias y la significatividad para dos de los tres atributos indican que las empresas que disponen de intranet presentan una mayor estabilidad de la plantilla, un menor estrés laboral en su personal y un efecto positivo en la reducción del absentismo. En definitiva, favorece el logro de mejores resultados en la satisfacción de los recursos humanos de la organización.

\subsection{Efecto de la intranet sobre los procesos de gestión del conocimiento}

El conocimiento es considerado como un recurso clave, y una de las utilidades atribuidas a las intranets es precisamente la de facilitar su gestión. Desde una perspectiva organizativa son un elemento fundamental del capital estructural (Sveiby, 1998; Harvey; Lusch, 1999; Tiwana, 2002; Sher; Lee, 2004), componente

\begin{tabular}{|c|l|c|c|c|c|c|c|c|}
\hline Adq1 & $\begin{array}{l}\text { Adquiere conocimiento a través de sus relaciones con otras } \\
\text { empresas del sector, universidad, centros tecnológicos, etc. }\end{array}$ & 1 & 2 & 3 & 4 & 5 & 6 & 7 \\
\hline $\boldsymbol{A d q 2} \mathbf{2}$ & $\begin{array}{l}\text { Se relaciona con profesionales y técnicos expertos externos a } \\
\text { su empresa. }\end{array}$ & 1 & 2 & 3 & 4 & 5 & 6 & 7 \\
\hline $\boldsymbol{A d q 3}$ & Acuden habitualmente a ferias y exposiciones. & 1 & 2 & 3 & 4 & 5 & 6 & 7 \\
\hline $\boldsymbol{A d q 4} \mathbf{4}$ & $\begin{array}{l}\text { El conocimiento procede del interior de la propia empresa. } \\
\text { Aprovechamos nuestra información procedente de } \\
\text { sugerencias y quejas de clientes, sugerencias del personal, } \\
\text { etc. }\end{array}$ & 1 & 2 & 3 & 4 & 5 & 6 & 7 \\
\hline
\end{tabular}

Tabla 5. Atributos de adquisición de conocimiento. Señale para cada pregunta el valor que mejor refleja la situación de su empresa, siendo $1=$ total desacuerdo y $7=$ total acuerdo

\begin{tabular}{|c|c|c|c|c|c|}
\hline $\begin{array}{c}\text { Utilización de } \\
\text { la intranet }\end{array}$ & \multicolumn{2}{|c|}{ (Medias) $A d q 1$} & Adq2 & Adq3 & Adq4 \\
\hline Sí & \multirow{2}{*}{\multicolumn{2}{|c|}{$\begin{array}{l}5,00 \\
2,75\end{array}$}} & 5,84 & 4,76 & 5,92 \\
\hline No & & & 3,50 & 4,25 & 5,50 \\
\hline & Valor de la $F$ & 9,25 & 15,37 & 0,47 & 0,35 \\
\hline & Significatividad & $0,007^{*}$ & $0,001^{*}$ & 0,501 & 0,556 \\
\hline & \multicolumn{5}{|c|}{ * Significatividad al nivel del $99 \%$} \\
\hline
\end{tabular}

Tabla 6. Anova posesión de intranet y atributos de la adquisición de conocimiento 
del capital intelectual que recoge el conocimiento que la organización consigue explicitar, sistematizar e interiorizar (Brooking, 1998; Stewart, 2001; Choo; Bontis, 2002; Adriessen, 2004; Truch; Edvinsson; Buzan, 2004).

De acuerdo con esto, resulta de interés analizar de forma empírica cómo afecta la utilización de intranet a cada una de las fases de la gestión del activo conocimiento: adquisición, distribución, interpretación y memoria, generando para cada una de estas etapas una escala likert basada en trabajos previos (Grant, 1996; Nonaka; Teece, 2001; Knowman-Project, 2003; Bueno; Ordóñez; Salmador, 2004; European Committee for Standardization, 2004), sobre la que los gerentes expresarían su opinión.

\subsubsection{Efecto de la intranet sobre la adquisición de conocimiento}

En primer lugar se analiza la existencia de relación entre la posesión de intranet y los atributos que componen la variable dependiente adquisición de conocimiento.

Como puede observarse en la tabla 6, esta posesión tiene un efecto positivo, resultando mayores niveles de actividad en los procesos de adquisición de conocimiento.

Cabe puntualizar que si bien todas las medias indican la mayor actividad en los procesos de adquisición de conocimiento de las empresas con intranet, la relación es estadísticamente significativa para dos atributos (Adq1 y $A d q 2$, adquisición de conocimiento procedente del exterior de la empresa) mientras que para la adquisición procedente del interior $(A d q 4)$ la relación no alcanza significatividad. En este caso concreto estamos ante un suceso similar al comentado para la relación entre intranet y satisfacción de los recursos humanos, donde si bien las medias indican mayor actividad de las empresas que poseen una, no se llega a alcanzar significatividad estadística posiblemente a causa del tamaño muestral.

\subsubsection{Efecto sobre la distribución de conoci- miento}

Los resultados de la tabla 8 muestran a nivel descriptivo que el grupo formado por las empresas que disponen de intranet presenta mayor actividad media en las actuaciones de distribución de conocimiento. Además, el contraste estadístico evidencia que existe un efecto directo, positivo y significativo sobre cada uno de sus atributos. En consecuencia, permite en mayor medida que los objetivos y estrategias de la organización sean conocidos por todo el personal, facilita la movilidad funcional de los empleados, el trabajo en equipo y favorece compartir las mejores prácticas por toda la organización.

\begin{tabular}{|c|l|c|c|c|c|c|c|c|}
\hline Dis1 & $\begin{array}{l}\text { Los objetivos de la empresa son comunicados a todos sus } \\
\text { miembros. }\end{array}$ & 1 & 2 & 3 & 4 & 5 & 6 & 7 \\
\hline Dis2 & $\begin{array}{l}\text { Se elaboran periódicamente informes escritos que se } \\
\text { reparten a todo el personal informando de los avances y } \\
\text { marcha de la empresa. }\end{array}$ & 1 & 2 & 3 & 4 & 5 & 6 & 7 \\
\hline Dis3 & $\begin{array}{l}\text { Existen en la organización empleados que participan en } \\
\text { varios equipos y actúan como enlaces. }\end{array}$ & 1 & 2 & 3 & 4 & 5 & 6 & 7 \\
\hline Dis4 & $\begin{array}{l}\text { Se dispone de mecanismos que garantizan que las mejores } \\
\text { prácticas son compartidas entre las distintas áreas de la } \\
\text { empresa. }\end{array}$ & 1 & 2 & 3 & 4 & 5 & 6 & 7 \\
\hline Dis5 & $\begin{array}{l}\text { Para distribuir información se utilizan las TIC, correo } \\
\text { electrónico, web, intranet. }\end{array}$ & 1 & 2 & 3 & 4 & 5 & 6 & 7 \\
\hline
\end{tabular}

Tabla 7. Atributos de distribución de conocimiento. Señale para cada pregunta el valor que mejor refleja la situación de su empresa, siendo $l=$ total desacuerdo y $7=$ total acuerdo.

\begin{tabular}{|c|c|c|c|c|c|c|}
\hline $\begin{array}{c}\text { Utilización de } \\
\text { la intranet }\end{array}$ & \multicolumn{2}{|c|}{ (Medias) Dis1 } & Dis2 & Dis3 & Dis4 & Dis5 \\
\hline Sí & \multicolumn{2}{|l|}{5,76} & 4,38 & 5,61 & 5,61 & 6,48 \\
\hline \multirow[t]{3}{*}{ No } & \multicolumn{2}{|l|}{3,12} & 2,00 & 3,37 & 1,75 & 3,12 \\
\hline & Valor de la $F$ & 13,73 & 10,08 & 10,50 & 84,81 & 29,23 \\
\hline & Significatividad & $0,002 *$ & $0,005 *$ & $0,004^{*}$ & $0,000 *$ & $0,000 *$ \\
\hline
\end{tabular}

Tabla 8. Anova posesión de intranet y atributos de la distribución de conocimiento 


\begin{tabular}{|c|l|l|l|l|l|l|l|l|}
\hline Int1 & $\begin{array}{l}\text { Todos los miembros de la organización perciben un } \\
\text { mismo propósito con el que se sienten comprometidos. }\end{array}$ & 1 & 2 & 3 & 4 & 5 & 6 & 7 \\
\hline Int2 & Los empleados comparten experiencias entre sí. & 1 & 2 & 3 & 4 & 5 & 6 & 7 \\
\hline Int3 & $\begin{array}{l}\text { Los empleados comprenden las funciones de otras } \\
\text { personas o departamentos. }\end{array}$ & 2 & 3 & 4 & 5 & 6 & 7 \\
\hline Int4 & $\begin{array}{l}\text { El trabajo en equipo es una práctica habitual en la } \\
\text { empresa. }\end{array}$ & 1 & 2 & 3 & 4 & 5 & 6 & 7 \\
\hline Int5 & $\begin{array}{l}\text { Utilizan las TIC para compartir experiencias, aprender } \\
\text { otros puestos, trabajar en equipo. }\end{array}$ & 2 & 3 & 4 & 5 & 6 & 7 \\
\hline
\end{tabular}

Tabla 9. Atributos de interpretación de conocimiento. Señale para cada pregunta el valor que mejor refleja la situación de su empresa, siendo $1=$ total desacuerdo y $7=$ total acuerdo.

\begin{tabular}{|c|c|c|c|c|c|c|}
\hline $\begin{array}{l}\text { Utilización de } \\
\text { la intranet }\end{array}$ & \multicolumn{2}{|c|}{ (Medias) Int1 } & Int2 & Int3 & Int4 & Int5 \\
\hline Sí & \multicolumn{2}{|l|}{5,61} & 5,92 & 5,38 & 6,15 & 6,46 \\
\hline \multirow[t]{4}{*}{ No } & \multicolumn{2}{|l|}{3,12} & 4,25 & 3,62 & 4,37 & 4,37 \\
\hline & Valor de la $F$ & 20,87 & 8,12 & 7,48 & 8,87 & 9,50 \\
\hline & Significatividad & $0,000 *$ & $0,01 * *$ & 0,130 & $0,008^{*}$ & $0,006^{*}$ \\
\hline & \multicolumn{6}{|c|}{$\begin{array}{l}\text { * Significatividad al nivel del } 99 \% \\
* * \text { Significatividad al nivel del } 95 \%\end{array}$} \\
\hline
\end{tabular}

Tabla 10. Anova posesión de intranet y atributos de la interpretación de conocimiento

\subsubsection{Efecto sobre la interpretación de conoci- miento}

La tabla 10 muestra cómo también se obtiene una relación positiva y estadísticamente significativa entre disponer de una intranet y todos los atributos, de forma que las empresas que la poseen presentan a su vez una mejor interpretación del conocimiento organizativo.

En definitiva, contribuye a que los miembros de la organización perciban un mismo propósito y misión, que se compartan experiencias entre el personal y se comprendan mejor las funciones desarrolladas por otros puestos y áreas de trabajo.

\subsubsection{Efecto sobre la memoria de conocimiento}

Una de las fases más importantes del proceso de gestión de conocimiento es la memoria o almacenamiento del conocimiento, individual y colectivo, para su reutilización continua.

\section{«Con una intranet mejoran los resultados de los recursos humanos»}

La tabla 12 muestra que disponer de intranet genera una mayor actividad media en todas las actividades dirigidas al almacenamiento del conocimiento. Además, la relación positiva es estadísticamente significativa para cuatro de los atributos. Por consiguiente la in- tranet facilita el almacenamiento de conocimiento individual y colectivo y su localización dentro de la organización, mediante directorios de personas según su área de actividad o conocimiento y el acceso a la documentación agrupada por contenidos.

\subsection{Efecto sobre los costes de coordinación}

Por último, en cuanto a las variables de tipo económico, son numerosos los autores que afirman que de su uso organizativo cabe esperar que se derive, entre otros, una mayor eficacia y eficiencia en la coordinación de la organización (Kaplan; Norton, 1992; Van Nievelt; Willcocks, 1997; Maggiolini; Salvador, 2002; Brynjolfsson; Hitt, 2003). De la misma opinión son los gerentes de las empresas encuestadas, quienes consideran que las intranets cumplen entre sus funciones principales ser tecnologías de coordinación, al permitir una gestión más eficaz de la información necesaria para el desarrollo de los procesos de negocio.

Se trata por tanto de analizar si contribuyen efectivamente a la disminución de los costes de coordinación internos, que representan todos los costes en los que incurre una organización por su funcionamiento excluidos los de producción y operaciones (Strassmann, 1997; Miñana, 2001).

El análisis se realiza como en los casos anteriores mediante un modelo anova, en el que la variable independiente representa los dos grupos: empresas con intranet y empresas sin, y como variable dependiente los costes de coordinación internos, en su evolución entre los años 2001 y 2004. 
El estudio del contraste (tabla 13) pone de manifiesto la existencia de relación favorable y significativa entre la posesión de intranet y la disminución de costes de coordinación internos.

\section{Conclusiones}

La medición de la rentabilidad y beneficios que la intranet genera en una organización es un tema complejo que llega a convertirse para las empresas en una barrera a la inversión en dichas tecnologías. La valoración generalmente se realiza mediante la utilización exclusiva de instrumentos financieros basados en individualizar y cuantificar los beneficios generados por la inversión. Sin embargo, esto se muestra insuficiente ante este tipo de inversiones, cuya actividad es apoyar todos los procesos de la organización, lo que dificulta individualizar sus beneficios, la mayoría de los cuales son de base intangible: compartir conocimiento, disponer de información a tiempo, etc., y por tanto, difícilmente cuantificables monetariamente. La combina- ción de mediciones económicas, junto con otras cualitativas generadas mediante indicadores de los aspectos que las propias organizaciones consideran críticos para su gestión, tales como recursos humanos, conocimiento, etc., se muestran como una metodología válida para obtener una visión más real de los verdaderos beneficios que una intranet puede generar en una organización.

En concreto, para el caso de 80 pymes del sector TIC y en relación con el reconocimiento que hacen de los recursos humanos y del conocimiento como factores críticos para el éxito de la organización, se analiza qué efectos genera en dichos factores cualitativos, y las consecuencias sobre una variable económica: los costes de coordinación internos. Los resultados demuestran que respecto a los recursos humanos la intranet es una herramienta eficaz para mejorar la satisfacción y motivación de los empleados. En cuanto al segundo, facilita todas y cada una de las fases que componen la gestión del conocimiento. Así, permite un

Tabla 11. Atributos de memoria de conocimiento. Señale para cada pregunta el valor que mejor refleja la situación de su empresa, siendo $1=$ total desacuerdo y $7=$ total acuerdo. El conocimiento útil para la toma de decisiones está almacenado en:

\begin{tabular}{|c|l|l|l|l|l|l|l|l|}
\hline Mem1 & Personas expertas, experiencias, anécdotas, opiniones. & 1 & 2 & 3 & 4 & 5 & 6 & 7 \\
\hline Mem2 & Manuales de procedimiento, documentación y archivos. & 1 & 2 & 3 & 4 & 5 & 6 & 7 \\
\hline Mem3 & $\begin{array}{l}\text { Bases de datos o intranet que permiten almacenar } \\
\text { experiencias y conocimientos para ser usados con } \\
\text { posterioridad. }\end{array}$ & 1 & 2 & 3 & 4 & 5 & 6 & 7 \\
\hline Mem4 & $\begin{array}{l}\text { Existen directorios de teléfonos o correos electrónicos por } \\
\text { áreas funcionales que permiten identificar al experto en un } \\
\text { tema concreto. }\end{array}$ & 1 & 2 & 3 & 4 & 5 & 6 & 7 \\
\hline Mem5 & Los empleados utilizan con frecuencia las bases de datos. & 1 & 2 & 3 & 4 & 5 & 6 & 7 \\
\hline
\end{tabular}

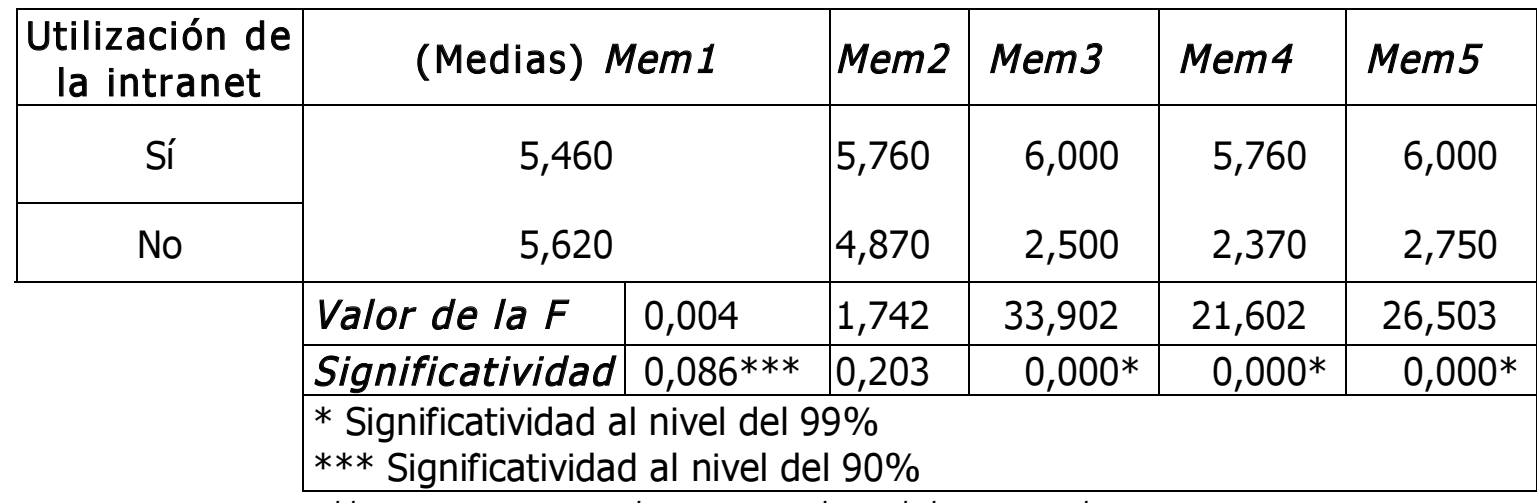

Tabla 12. Anova posesión de intranet y atributos de la memoria de conocimiento

\begin{tabular}{|c|c|c|}
\hline $\begin{array}{l}\text { Utilización de } \\
\text { la intranet }\end{array}$ & \multicolumn{2}{|c|}{ Media "costes de coordinación" } \\
\hline Sí & \multicolumn{2}{|l|}{$-0,006$} \\
\hline No & \multicolumn{2}{|l|}{0,330} \\
\hline & Valor de la $F$ & 2,900 \\
\hline & Significatividad & $0,092 * * *$ \\
\hline & \multicolumn{2}{|c|}{ *** Significatividad al nivel del $90 \%$} \\
\hline
\end{tabular}

Tabla 13. Anova de la posesión de intranet y costes de coordinación internos 
mayor nivel de actividad en las tareas conducentes a adquirir conocimiento, facilita su distribución por toda la organización, logra una interpretación y comprensión más uniforme de los objetivos y estrategias de la empresa, y facilita notablemente el almacenamiento de conocimiento, ya sea individual o colectivo. Por último, además de en los indicadores cualitativos, también se obtienen resultados económicos positivos, al contribuir a reducir en el tiempo los costes de coordinación internos de la organización.

Agradecimientos: este trabajo ha sido posible gracias a la financiación pública obtenida mediante el Plan Nacional I+D+I: DPI2002Q4342-C05-5.

\section{Notas}

1. Investigación realizada dentro del proyecto Cicyt (Comisión Interministerial de Ciencia y Tecnología), con referencia DPI2002Q4342-C05-5, titulado: Diseño e implantación de un sistema de ayuda a la toma de decisiones para la gestión de la empresa.

2. Definición establecida por la Comisión Europea (DOCE 20.05.2003), oficial a partir del 1 de enero del 2005, que se resume a continuación:

Microempresa: menos de 10 trabajadores y un límite de 2 millones de euros para el volumen de negocios y el balance general.

Pequeñas empresas: entre 10 y 49 empleados. El límite del volumen de negocios y el del balance general es de 10 millones de euros.

Medianas empresas: entre 50 y 249 trabajadores. El límite del volumen de negocios será de hasta 50 millones de euros, y el del balance general, hasta 43 millones de euros.

3. Instrumento estadístico de medición ordinal consistente en una serie de ítems o juicios, referentes a un mismo concepto, ante los cuales se solicita la opinión del sujeto encuestado (Sánchez; Sarabia, 1999). Hacen posible medir actitudes y comportamientos.

4. El Alpha de Cronbach es un método estadístico basado en la correlación promedio de las variables o ítemes que componen cada uno de los conceptos. Nos indica si la escala es fiable y por tanto libre de error aleatorio, considerándose satisfactorios los niveles de alfa superiores a 0,7

(Hair, et al., 1999). En esta investigación el valor mínimo que se obtuvo fue de 0,755 . Por tanto, superior al valor de corte por encima del cual se considera una escala fiable.

5. Analysis of variance. Modelos estadísticos de análisis de la varianza que permiten comprobar la potencia explicativa de un factor, variable independiente de tipo categórico, sobre una variable dependiente de carácter métrico. Las técnicas anova se emplean para contrastar la significación estadística de las diferencias de medias entre grupos o niveles de la variable independiente y ayudan a establecer estadísticamente la existencia de relaciones entre variables, así como la dirección o efecto que toma dicha relación (Hair, et al., 1999).

6. Estadístico que permite comprobar la existencia de diferencias estadísticamente significativas en las varianzas de dos poblaciones normales.

7. Las relaciones son estadísticamente significativas en función del valor que tome la significatividad, también llamada " $p$-valor". Cuando el valor de la significatividad sea inferior a 0,10 la relación será estadísticamente significativa a un nivel de confianza del $90 \%$. Siendo lo más favorable que el nivel de confianza sea lo más alto posible, del 95\% cuando la significatividad es menor que 0,05 y del $99 \%$ cuando toma valor menor de 0,01 .

\section{Bibliografía}

Andriessen, D. «IC valuation and measurement: classifying the state of the art». En: Journal of intellectual capital, 2004, v. 5, n. 2, pp. 230-242.

Brooking, A. «Intellectual capital: an exploratory study that develops measures and models». En: Management decision, 1998, v. 36, n. 2, pp. $67-76$.
Brynjolfsson, E.; Hitt, L. "Computing productivity: firm level evidence". En: Review of economics and statistics, 2003, noviembre, n. 85, pp. 339-376.

Brynjolfsson, E.; Hitt, L. "Paradox lost? Firm-level evidence on the returns to information systems spending". En: Management science, 1996, v. 42, n. 4 , pp. $541-558$.

Bueno, E.; Ordóñez, P.; Salmador, M. P. "Towards an integrative model of business, knowledge and organisational learning processes". En: International journal technology management, 2004, v. 27, n. 6, pp. 562-575.

Cañibano, L. "Información sobre capital intelectual: nueva iniciativa en la Unión Europea”. En: AECA: Revista de la Asociación Española de Contabilidad y Administración de Empresas, 2005, n. 72, pp. 77-80.

Cañibano, L.; Sánchez, P. «Medición, gestión e información de intangibles: lo más nuevo». En: Contabilidad y dirección, 2004, n. 1, pp. 99-139.

Chen, Y.; Liang, L.; Yang, F.; Zhu, J. "Evaluation of information technology investment: a data envelopment analysis approach". En: Сотрuters and operations research, 2006, mayo, v. 33, n. 5, pp. 1.368-1.379.

Choo, C. W.; Bontis, N. The strategic management of intellectual capital and organizational knowledge. Oxford: Oxford University Press, 2002, ISBN 019515486X

Comisión Europea. i2010-Una sociedad de información europea para el crecimiento y el empleo. Consultado en: 18-05-05.

http://europa.eu.int/information_society/eeurope/i2010/index_en.htm

Commission of the European Communities (COM). Communication from the Commision to the Council and the European Parliament; eEurope 2005: Benchmarking Indicators. Brussels, 21-11-02 COM(2002).

Dirección General de Política de la Pyme, (Dgpyme). Estadísticas Pyme. Consultado en: 03-05-05.

http://www.ipyme.org/IPYME/es-ES/EstadisticasPublicacionesEstudios/

EITO, European Information Technology Observatory. European Information Technology Observatory 2005. Consultado en: 17-05-05. http://www.eito.com/index-eito.html

European Committee for Standardization (CEN). European guide to good practice in knowledge management. Brussels, 2004, marzo. CWA 14924-5.

http://www.cenorm.be/cenorm/businessdomains/businessdomains/isss/cwa /knowledge+management.asp

Escobar, B. La evaluación económica de los sistemas de información. Ed. Universidad de Sevilla, 1997, ISBN 84-472-0345-X.

Grant, R. M. «Toward a knowledge-based theory of the firm». En: Strategic management journal, 1996, v. 17, número especial de invierno, pp. 109-122.

Hair, J. F.; Anderson, R. E.; Tatham, R. L.; Black, W. C. Análisis multivariante. Madrid: Prentice Hall, 1999, ISBN 8483220350.

Harvey, M. G.; Lusch, R. F. "Balancing the intellectual capital books: intangible liabilities". En: European management journal, 1999, February, v. 17 , n. 1 , pp. $85-92$.

http://knowman.ifw.uni-bremen.de/impressum.es.htm

Im, K. S.; Dow, K. E.; Grover, V. "Research report: a reexamination of IT investment and the market value of the firm. An event study methodology". En: Information systems research, 2001, March, v. 12, n. 1, pp. 103-117.

Instituto Nacional de Estadística (INE). Encuesta sobre el uso de las tecnologías de la información y las comunicaciones y del comercio electrónico. Año 2001, 2002, 2003 y 2004. Consultado en: 02-05-06. http://www.ine.es/inebase/

Kaplan, R. S.; Norton, D. P. "The balanced scorecard. Measures that drive performance". En: Harvard business review, 1992, January-February, v. 70, n. 1, pp. 71-79.

Kaplan, R. S.; Norton, D. P. The strategy-focused organization. Boston: Harvard Business School Press, 2001, ISBN 1-57851-250-6.

Knowman-Project. Proyecto de gestión de conocimiento financiado por la Comisión Europea. Consultado en: 03-05-05.

http://knowman.ifw.uni-bremen.de/impressum.es.htm 


\section{Próximos temas centrales}

\begin{tabular}{|c|c|}
\hline Noviembre 2006 & Vigilancia tecnológica \\
\hline Enero 2007 & Ciencia y tecnología de la información (InSciT2006) \\
\hline Marzo 2007 & Web 2.0: blogs, participación y Lib 2.0 \\
\hline Mayo 2007 & Portales y gestión de contenidos (CMS) \\
\hline Julio 2007 & Bibliotecas universitarias \\
\hline Septiembre 2007 & Documentación multimedia \\
\hline Noviembre 2007 & Ontologías \\
\hline
\end{tabular}

Los interesados pueden remitir notas, artículos, propuestas, publicidad, comentarios, etc., sobre estos temas a: epi@elprofesionaldelainformacion.com

Lev, B.; Sarath, B.; Sougianis, T. "R\&D reporting biases and their consequences". Working paper, University of New York, 1999.

Lev, B. Intangibles: management, measurement and reporting. Washington, D. C.: Brookings Institution Press, 2001, ISBN 0-8157-0094-6.

Maggiolini, P.; Salvador-Valles, R. "Validez de un modelo basado en los costes de transacción para identificar los beneficios de los SIIO (sistemas de información interorganizativos)". En: Gestão \& produção, 2002, v. 9 , n. 3 , pp. $235-244$

Mahmood, M. A.; Mann, G. J. "Measuring the organizational impact of information technology investment: an exploratory study". En: Journal of management information systems, 1993, v. 10, n. 1, pp. 97-122.

Markus, R.; Watters, B. Collective knowledge: intranets, productivity, and the promise of the knowledge workplace. Redmond, Washington: Microsoft Press, 2002, ISBN 0735614997.

Miñana, J. L. Desarrollo de un modelo que permita el diagnóstico en la aportación de valor de la infraestructura de tecnologías de la información. Valencia: Tesis doctoral no editada, Universidad Politécnica de Valencia, 2001.

Nonaka, I.; Teece, D. J. "Research directions for knowledge management". En: Nonaka, I.; Teece, D. J. Managing industrial knowledge: creation, transfer and utilization. Londres: SAGE, 2001, pp. 330-335.

O'Brien, J. A. Sistemas de información gerencial. Traducido de la cuarta edición de Management information systems. Colombia: Irwin McGraw-Hill, 2001, ISBN 958-41-0177-3.

OCDE. Promoting entrepreneurship and innovative SMEs in a global economy. The second OECD ministerial conference on small and medium enterprises (SMEs). Consultado en: 08-07-05.

http://www.oecd-istanbul.sme2004.org

Ontiveros, E.; Manzano, D.; Rodriguez, I. Tecnologías de la información y las comunicaciones (TIC), crecimiento económico y actividad empresarial. Madrid: Círculo de empresarios, 2004, julio.

Paños, A. "Influencia de las tecnologías de la información en los procesos de información y toma de decisiones de las empresas". En: I Congreso universitario de ciencias de la documentación, teoría, historia y metodología de la documentación en España, 2000.

Peters, G. "Evaluating your computer investment strategy". En: Journal of information technology, 1990, v. 2, pp. 197-208.

Powelt, T.; Dent-Micallef, A. "Information technology as competitive advantage: the role of human, business and technology resources". En: Strategic management journal, 1997, v. 18, n. 5, pp. 375-405.
Red.es y Ministerio de industria, turismo y comercio. La microempresa española en la sociedad de la información. Consultado en: 07-06-05. http://observatorio.red.es/estudios/comercio/index.html

Sánchez, M.; Sarabia, F. J. "Validez y fiabilidad de escalas". En: Sarabia, F. J. Metodología para la investigación en marketing y dirección de empresas. Madrid: Pirámide, 1999, pp. 361-392, ISBN 84-368-1342-1.

Sher, P.; Lee, V. C. "Information technology as a facilitator for enhancing dynamic capabilities through knowledge management". En: Information and management, 2004, noviembre, v. 41, n. 8, pp. 933-945.

Sieber, S.; Valor, J. Las TIC como agente de cambio en la empresa española. Situación actual y tendencias de futuro. Consultado en: 11-07-05. http://www.iese.edu/es/files/5_15975.pdf

Standfield, K. Intangible management: tools for solving the accounting and management crisis. Orlando: Academic Press Inc, 2002, ISBN 0126633517.

Stewart, T. A. Intellectual capital: the new wealth of organizations. London: Nicholas Brearley, 1997, ISBN 1-85788-183-4.

Stewart, T. A. The wealth of knowledge: intellectual capital and the twenty-first century organization. New York: Doubleday, 2001, ISBN 0385500718 .

Strassman, P. A. The squandered computer: evaluating the business alignment of information technologies. New Canaan: Information Economics Press, 1997, ISBN 0962041319.

Sveiby, K. The new organizatonial wealth: managing and measuring intangible assets. San Francisco: Berret-Koelher Publishers, 1998, ISBN 1-57675-014-0.

Tissen, R.; Andriessen, D.; Lekanne, F. El valor del conocimiento para aumentar el rendimiento en las empresas. Madrid: Prentice Hall. 2000.

Truch, E.; Edvinsson, L.; Buzan, T. Leveraging corporate knowledge. Maimi: Caiman, 2004, ISBN 0566085763.

Tiwana, A. The knowledge management toolkit: practical techniques for building a knowledge management system. Upper Saddle River, NJ: Prentice Hall, 2002, ISBN 013009224X.

Van Nievelt, M. C. A.; Willcocks, L. «Benchmarking organisational \& IT performance». En: Oxford executive research briefings, 1997, n. 6.

Daniel Pérez González, Pedro Solana González, Facultad de Ciencias Económicas y Empresariales. Universidad de Cantabria, Santander. daniel.perez@unican.es pedro.solana@unican.es 